Please do not remove this page

RMIT

UNIVERSITY

\title{
Dynamical heterogeneity and the freezing transition in hard-sphere suspensions: Further analysis of the mean square displacement and the velocity autocorrelation function
}

Van Megen, William; Bryant, Gary

https://researchrepository.rmit.edu.au/esploro/outputs/9921860357801341/filesAndLinks?institution=61RMIT_INST\&index=null

Van Megen, W., \& Bryant, G. (2007). Dynamical heterogeneity and the freezing transition in hard-sphere suspensions: Further analysis of the mean square displacement and the velocity autocorrelation function. Physical Review E, 76(2), 021402-1-021402-021408. https://doi.org/10.1103/PhysRevE.76.021402

Published Version: https://doi.org/10.1103/PhysRevE.76.021402

Repository homepage: https://researchrepository.rmit.edu.au

(c) 2007 The American Physical Society

Downloaded On 2023/04/26 23:40:22 +1000 


\title{
Dynamical heterogeneity and the freezing transition in hard-sphere suspensions: Further analysis of the mean square displacement and the velocity autocorrelation function
}

\author{
W. van Megen and G. Bryant \\ Department of Applied Physics, Royal Melbourne Institute of Technology, Melbourne, Victoria 3000, Australia
}

(Received 23 May 2007; published 14 August 2007)

\begin{abstract}
The velocity autocorrelation function is derived from the mean-squared displacement measured on a colloidal suspension of particles with hard-sphere-like interactions. It decays to zero from below and follows a stretched exponential function of delay time for the thermodynamically stable suspension. For the metastable suspension a power-law decay emerges. The results are discussed in terms of the classical Lorentz gas and the model that describes diffusion confined to one dimension. With the aid of these models, the experimental results provide a characterization of the dynamical heterogeneities which are observed microscopically, and an explanation for the enhanced resistance to flow and diffusion usually found in undercooled fluids upon approaching the glass transition.
\end{abstract}

DOI: 10.1103/PhysRevE.76.021402

PACS number(s): 82.70.Dd, 64.70.Pf, 61.20.Ne

\section{INTRODUCTION}

Colloidal suspensions with hard-sphere-like interactions show a first order transition from colloidal fluid to an iridescent crystal [1] as well as a change in dynamical properties that is commonly identified with the glass transition [2]. The attraction of colloidal suspensions for the experimentalist is that crystallization generally occurs sufficiently slowly to be resolved in real time. Moreover, the nucleation rate, or lifetime of the metastable fluid suspension, is very sensitive to, and indeed can be controlled by, small changes in the distribution of particle radii $[3,4]$. On the other hand, the suspension's dynamics, as expressed by the time correlation function of the particle number density fluctuations, is comparatively insensitive to the details of the particle size distribution (PSD) [3]. This fortuitous influence of the PSD, or polydispersity, has been exploited, though not always intentionally, in measurements of crystallization kinetics and particle dynamics.

Of course the same applies to computer simulations of simple atomic fluids. A one-component fluid of ballistic hard spheres, for example, when only slightly undercooled-i.e., when its volume fraction exceeds the known freezing value, $\varphi_{f}=0.493$, by only a few percent—crystallizes too fast to calculate its dynamical and structural properties. Here too, polydispersity is introduced to delay nucleation $[5,6]$. However, since it is clearly advantageous to know the location of the equilibrium phase boundaries, whatever system is under investigation, this delay must not be indefinite. In very broad terms, a polydispersity between approximately 5 and $10 \%$ appears to satisfy these requirements.

Many recent studies on colloidal systems, of both hard spheres and particles with finite range interactions, have been directed toward gaining insight into the mechanism responsible for the glass transition [7]. Computer simulations [8] and optical microscopy [9-11] expose a picture of heterogeneous dynamics, particularly evident at the elevated particle number densities near the glass transition, in which the majority of particles are seen to be confined to domains or clusters that are separated by comparatively mobile interstitial particles. As the suspension's volume fraction is increased, the probability that a cluster percolates the system also increases. This, as shown recently, decreases the frequency of the shear stress that can be supported [11].

Recent dynamic light scattering $[12,13]$ and molecular dynamics studies [6] of colloidal and ballistic hard-sphere systems, respectively, expose qualitative differences between the thermodynamically stable $\left(\varphi<\varphi_{f}\right)$ and metastable $\left(\varphi>\varphi_{f}\right)$ fluids. In particular it was found that on traversing the freezing volume fraction, $\varphi_{f}$, from below, negative power-law tails develop in the velocity autocorrelation function. It was also argued that such power-law tails are incommensurate with purely viscous flow $[6,13]$.

So, by closing off avenues by which the material can flow, both microscopy and spectroscopy expose routes toward solidification. The question is whether there is any consistency between the two approaches. Can their respective results be buttressed so as to furnish a complete explanation of the glass transition? We attempt a step in this direction in this paper by analyzing the particles' non-Markovian displacement statistics, as manifested by the slow, negative decay of the velocity autocorrelation function and the stretching of the mean-squared displacement, in terms of the Lorentz gas [14] and a model that describes diffusing particles confined to one dimension [15].

\section{PROCEDURES AND THEORY}

Dynamic light scattering procedures and the preparation and characterization of colloidal particles are described extensively in previous work $[12,16,17]$, and are summarized here only briefly. The suspension comprises a mixture of polymer particles ( $98 \%$ by weight, polydispersity $\sim 7 \%$ ) and silica particles $(2 \%$ by weight, polydispersity $\sim 2 \%$ ) of the same average radius $(R=200 \mathrm{~nm})$. The polymer particles consist of a copolymer core of methylmethacrylate (MMA) and tri-fluoroethylacrylate (TFEA). This particular copolymer is chosen so that the refractive index of particles can be closely matched to a single suspending solvent, cis-decalin. The hard-sphere character for both types of particles is provided by a stabilizing coating of poly-12-hydroxystearic acid 
(PHSA), about $10 \mathrm{~nm}$ thick, bonded to the surface. Both phase diagrams and direct measurements of the force between the stabilizing layers [18] show that these particles behave as near perfect hard spheres.

The effective hard-sphere volume fractions, $\varphi$, of the samples were determined by equating the observed freezing volume fraction (determined by weight), with the known freezing value, $\varphi_{f}=0.494$, of the perfect hard-sphere system. This gives melting and glass transition volume fractions of $\varphi_{m}=0.535 \pm 0.005$ and $\varphi_{g}=0.565 \pm 0.005$, respectively. The volume fractions measured are in the range $0.16 \leqslant \varphi \leqslant \varphi_{g}$. In the undercooled samples, $\left(\varphi>\varphi_{f}\right)$ samples are tumbled for several hours prior to the measurements to break up any crystals which may be present. Measurements are made on a time scale of $1000 \mathrm{~s}$, long before there is evidence of any nucleation. This is demonstrated in several ways: (i) during the time of these measurements no Bragg reflections become evident by eye; (ii) the static structure factors show no discernable crystal-like features; (iii) in all cases the measurements are independent of the commencement time of the measurements [17] —in other words the measurements were made over a time window in which the suspension's properties are (quasi-) stationary.

Distances are expressed in units of the (average) particle radius, and delay times in units of the Brownian characteristic time, $\tau_{b}=R^{2} /\left(6 D_{o}\right)=0.021 \mathrm{~s}$. $D_{o}$ is the diffusion coefficient of a freely diffusing particle. The composition and refractive indices of the mixture are such that fluctuations in the laser light scattered from the particle number density fluctuations are entirely suppressed, so that the measured intermediate scattering function (ISF) equates with the selfISF, given by

$$
F_{s}(q, \tau)=\langle\exp [i \mathbf{q} \cdot \Delta \mathbf{r}(\tau)]\rangle,
$$

where $\mathbf{q}$ is the wave vector and $\Delta \mathbf{r}(\tau)$ the particle displacement in the time interval $\tau$. Measurement of $F_{s}(q, \tau)$ is detailed in other papers $[16,17]$.

Only the zero wave vector, or Gaussian, limit of the ISF

$$
F_{s}(q \rightarrow 0, \tau)=F_{s}^{(G)}(q, \tau)=\exp \left[-q^{2}\left\langle\Delta r^{2}(\tau)\right\rangle / 6\right]
$$

is considered here. $\left\langle\Delta r^{2}(\tau)\right\rangle$ is the particle mean-squared displacement (MSD). The other property of interest is the velocity autocorrelation function (VAF) which is defined by [19]

$$
Z(\tau)=-\lim _{q \rightarrow 0} \frac{1}{q^{2}} \frac{d^{2}}{d \tau^{2}} F_{s}(q, \tau)=\frac{d^{2}}{d \tau^{2}}\left\langle\Delta r^{2}(\tau)\right\rangle .
$$

There are several other quantities required for analysis and discussion of the data. The first is an index that gives the maximum degree of stretching of the MSD, calculated from the minimum value of the logarithmic slope of the MSD:

$$
\nu=\min \left[\frac{d \log _{10}\left\langle\Delta r^{2}(\tau)\right\rangle}{d \log _{10} \tau}\right] .
$$

We refer to this quantity as the stretching index. The delay time and root-mean-squared displacement (RMSD) where this occurs are designated as $\tau_{m}$ and $R_{m}$, respectively.

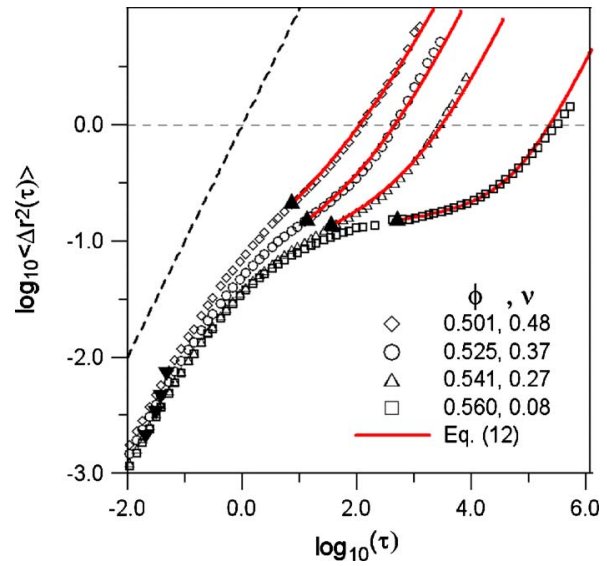

FIG. 1. (Color online) Double logarithm plot of the MSD vs delay time for volume fractions and stretching indices indicated. The lower and upper bold triangles denote the points $\left(R_{c}^{2}, \tau_{c}\right)$ and $\left(R_{m}^{2}, \tau_{m}\right)$, respectively. The heavy dashed line is the MSD for the ideal dilute suspension. The fits are made using Eq. (12). See text for further details.

Two other quantities are the short- and long-time selfdiffusion coefficients,

$$
D_{s}=\lim _{\tau \rightarrow 0} \frac{\left\langle\Delta r^{2}(\tau)\right\rangle}{\tau} \quad \text { and } \quad D_{l}=\lim _{\tau \rightarrow \infty} \frac{\left\langle\Delta r^{2}(\tau)\right\rangle}{\tau},
$$

where the limits " 0 " and " $\infty$ " mean the lower $\left(\sim 10^{-3} \mathrm{~s}\right)$ and upper $\left(\sim 10^{3} \mathrm{~s}\right)$ limits of the experimental time window. Finally, we will make reference to the average gap

$$
R_{c}=\left(\frac{\phi_{R}}{\phi}\right)^{1 / 3}-1
$$

between two particles, where $\varphi_{R}=0.64$ is the volume fraction at random close packing. Given $R_{c}$, the average interval, $\tau_{c}$, between diffusive particle encounters is then read from the MSD.

\section{RESULTS}

Some of the results shown below have been reported previously $[12,13]$, but they are shown here for completeness and because they are required for further analysis below. Typical MSDs are shown in Fig. 1. Points $\left(\tau_{c}, R_{c}^{2}\right)$, indicating the time and mean-squared distance between particle encounters, and $\left(\tau_{m}, R_{m}^{2}\right)$, where the logarithmic slope of the MSD is a minimum, are also shown. This minimum, or stretching index, $\nu$, is set out against volume fraction, $\varphi$, in Fig. 2. Note that $\nu\left(\varphi_{f}\right) \approx 1 / 2$ and $\nu\left(\varphi \rightarrow \varphi_{g}\right)=0$. Figure 3 shows the variation of $\tau_{m}, \tau_{c}$ and the ratio $\tau_{m} / \tau_{c}$ with $\varphi$, and Fig. 4 the variation of $R_{m}, R_{c}$ and the ratio $R_{m} / R_{c}$ with $\varphi$. The ratios $\tau_{m} / \tau_{c}$ and $R_{m} / R_{c}$ express the time and length scales that characterize the collective, non-Markovian processes in the suspension relative to the time and length scales that characterize the diffusion of particles in their neighbor cages. The latter process is also characterized by the short-time diffusion coefficient, $D_{s}=R_{c}^{2} / \tau_{c}$ [20].

For volume fractions less than about 0.2 , stretching of the MSDs is weak and the VAFs, calculated from them by Eq. 


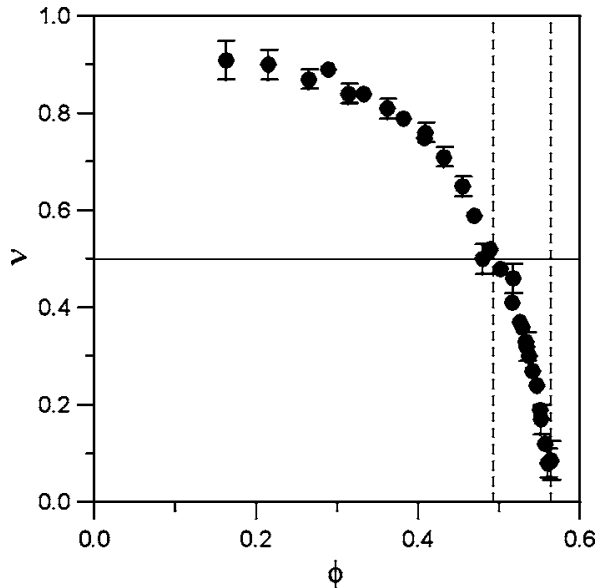

FIG. 2. Stretching index, $\nu$, defined in Eq. (4), vs volume fraction. The dashed vertical lines are located at the freezing and glass transition volume fractions $\varphi_{f}=0.493$ and $\varphi_{g}=0.565$. These lines are shown in all figures where $\varphi$ is the abscissa.

(3), apart from being negative, contain little systematic information. As shown in Ref. [13], at higher volume fractions minima can generally be discerned at around $\tau=10^{-2}$. Beyond these minima the VAFs decay monotonically to the noise floor from below-there is no indication of $Z(\tau)$ becoming positive as the upper limit of the experimental time window is approached. Our focus here is on just this slow negative decay shown by way of double logarithm plots of $|Z(\tau)|$ versus $\tau\left(>10^{-2}\right)$ in Fig. 5(a), for the thermodynamically stable suspension $\left(\varphi<\varphi_{f}\right)$ and in Fig. 5(b) for the metastable suspension $\left(\varphi>\varphi_{f}\right)$.

For the thermodynamically stable suspension the VAFs show no systematic variation with volume fraction and they are seen to be compatible with the stretched exponential,

$$
Z\left(\tau>\tau_{v}\right)=-B \exp \left[-\left(\frac{\tau}{\tau_{d}}\right)^{\zeta}\right]
$$

with $B=0.5 \pm 0.1, \quad \zeta=0.3 \pm 0.02$, and $\tau_{d}=1.0( \pm 0.3) \times 10^{-3}$. This is not the case for higher volume fractions, $\varphi>\varphi_{f}[$ Fig.

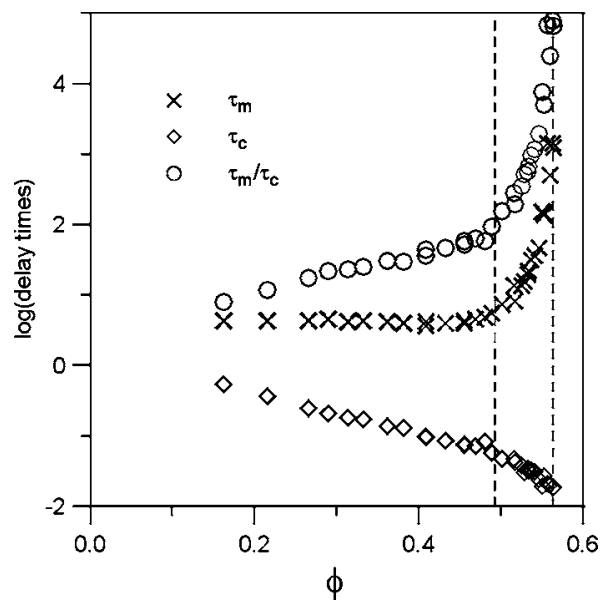

FIG. 3. Logarithm of characteristic delay times vs volume fraction. See text for further details.

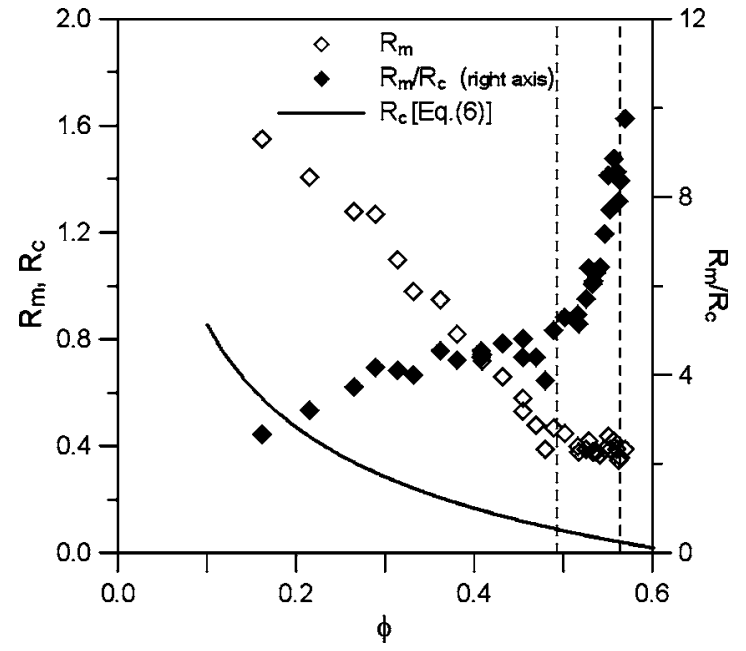

FIG. 4. Characteristic displacements vs volume fraction. See text for further details

5(b)]. Significant deviations from Eq. (7) are apparent, even with allowance for errors in the parameters and noise on the data. As is illustrated in Fig. 5(c), for a volume fraction ( $\varphi=0.533)$ about midway between $\varphi_{f}$ and $\varphi_{g}$, only the initial part of the decay of the VAF is compatible with a stretched exponential. Beyond $\tau \approx 1$ it appears that the power law

$$
Z(\tau \geqslant 1)=-\left(\frac{\tau}{\tau_{f}}\right)^{-\mu}
$$

provides a better description of the data. The index $\mu$ of the best fitting power laws to the data, from an arbitrary lower limit of $\log _{10}(\tau)=0$, is shown in Fig. 6. Outside the considerable uncertainties, the line of best fit to $\mu$ versus $\varphi$ suggests a systematic increase from $\mu \approx 1.5$, at $\varphi_{f}$, to $\mu \approx 2$, at $\varphi_{g}$. The quantity $2-\nu$ is also plotted in Fig. 6 . The fitting of straight lines to the indices $\mu$ and $2-\nu$ is not meant to imply either of them depends linearly on $\varphi$. The scaling time $\tau_{f}$ (shown in Fig. 7) shows no variation with volume fraction, and yields a value of $\log _{10}\left(\tau_{f}\right)=-1.2 \pm 0.3$. The other quantities in Fig. 7 are discussed below.

\section{DISCUSSION AND ANALYSES}

\section{A. Consistency checks}

Whatever the nature of the dynamical mode, characterized by the algebraic decay in the VAF (Fig. 5), it emerges around the first order transition point, $\varphi_{f}$. Note also, in Fig. 3, that around the same volume fraction the delay time $\tau_{m}$ starts to increase and, from Fig. 4, that the RMS displacement at $\tau_{m}$ saturates. Of course, experimental noise and the limited resolution in $\varphi$ does not allow us to infer categorically the occurrence of a qualitative change in the particle dynamics at $\varphi_{f}$ precisely. In this respect a more definitive inference can be drawn from molecular dynamics studies of ballistic hard spheres [6].

In view of the potential implications of the above results with regard to the dynamics of the first order freezing transition, we perform a number of consistency checks and, by 

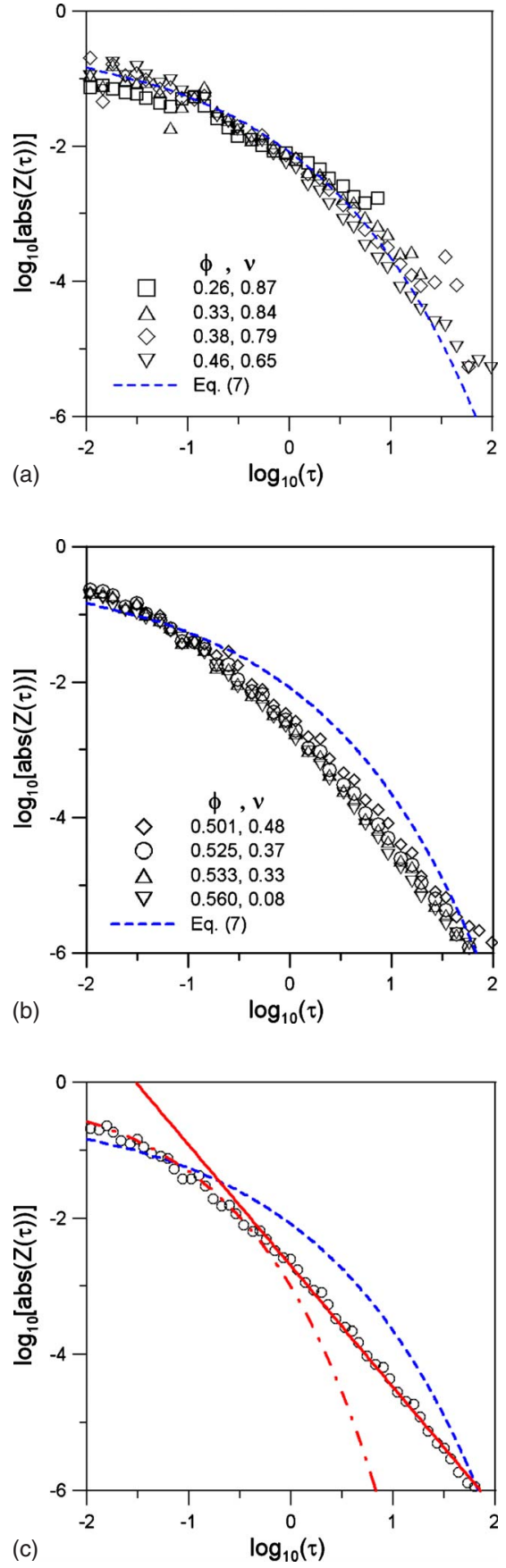

FIG. 5. (Color online) Logarithm of the modulus of the VAF vs logarithm of delay time: (a) For values of $\varphi\left(<\varphi_{f}\right)$ and corresponding values of $\nu(>1 / 2)$ indicated; (b) values of $\varphi\left(>\varphi_{f}\right)$ and corresponding values of $\nu(<1 / 2)$ indicated; (c) for $\varphi=0.533$. The dashed line in all cases is the stretched exponential [Eq. (7)] that best describes the VAFs for $\varphi<\varphi_{f}$. The dashed-dotted line in (c) is the stretched exponential that passes through $Z(\tau)$ for $\tau<1$. The solid line in (c) is the power law [Eq. (8)] that passes through $Z(\tau)$ for $\tau>1$.

means of these, see in what other ways the thermodynamically stable and metastable suspensions can be differentiated. We begin by examination of the power-law decay of the VAF. The data shown in Fig. 5(b), obtained by double differentiation of the MSDs, have suffered significant noise am-

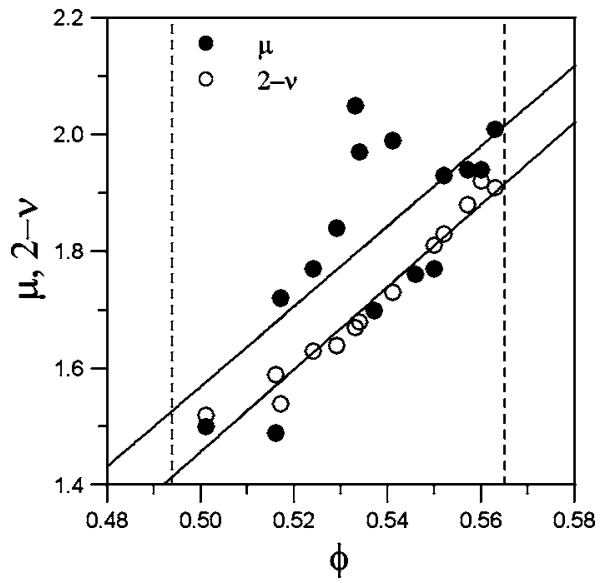

FIG. 6. Exponent $\mu$ of the power law, Eq. (8), and $2-\nu$, where $\nu$ is the stretching index defined in Eq. (4), vs volume fraction. The upper and lower lines are lines of best fit to $\mu$ and $2-\nu$.

plification. This is reflected in the noise on the parameters, $\mu$ and $\tau_{f}$, that characterize the power law. An alternative approach is to calculate the MSD, according to Eq. (3), by double integration of $Z(\tau)$ by assuming, for $\tau \geqslant \tau_{m}$ and $\varphi$ $>\varphi_{f}$, that $Z(\tau)=-K \tau^{-\mu}$, where $K$ is a constant. This gives the following general expression for the MSD:

$$
\left\langle\Delta r^{2}\left(\tau \geqslant \tau_{m}\right\rangle=\left[-K \frac{\tau^{2-\mu}}{(1-\mu)(2-\mu)}+K^{\prime} \tau+K^{\prime \prime}\right],\right.
$$

provided $\mu \neq 2$ [21]. By our definition $\left\langle\Delta r^{2}\left(\tau_{m}\right)\right\rangle=R_{m}^{2}$. Thus it is convenient to rewrite the MSD in terms of $R_{m}$ and $\tau_{m}$ :

$$
\left\langle\Delta r^{2}\left(\tau \geqslant \tau_{m}\right)\right\rangle=\left[C\left(\frac{\tau}{\tau_{m}}\right)^{\delta}+C^{\prime}\left(\frac{\tau}{\tau_{m}}\right)+C^{\prime \prime}\right] R_{m}^{2},
$$

where $\delta=2-\mu$ and $C+C^{\prime}+C^{\prime \prime}=1$ by definition. Another boundary condition comes from the logarithmic slope of the MSD:

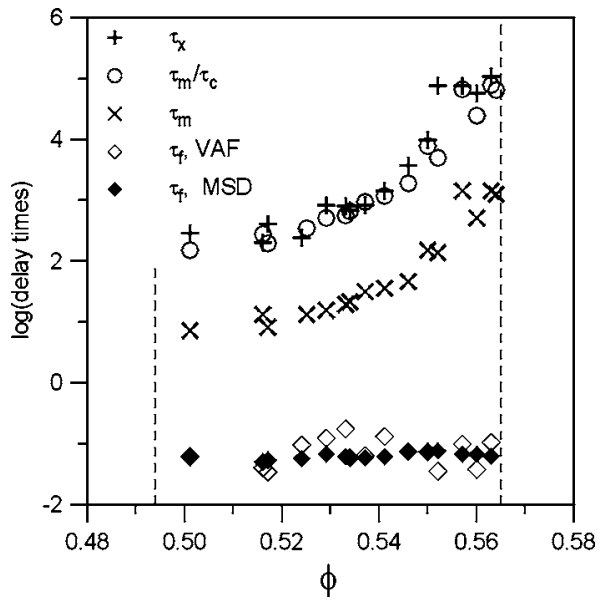

FIG. 7. Logarithm of characteristic delay times vs volume fraction. The open diamonds represent values of $\tau_{f}$ obtained by fitting power laws directly to the VAFs, whereas the closed diamonds are obtained by the analysis with Eq. (12). See text for further details. 


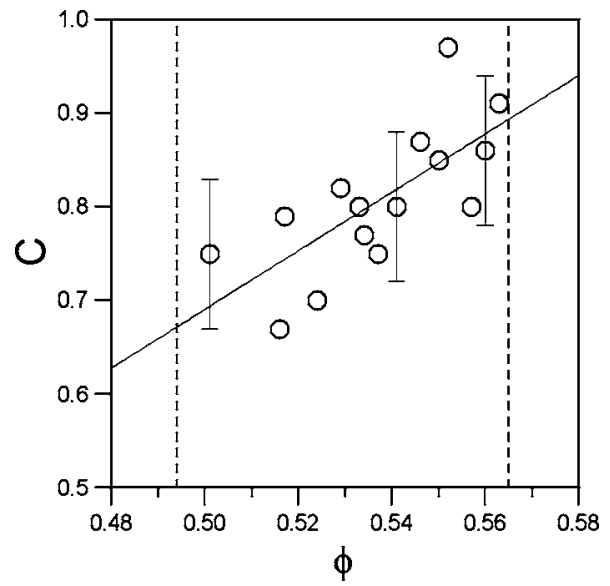

FIG. 8. Coefficient of the first term in Eq. (12) vs volume fraction. The solid line is the line of best fit. Typical error bars are shown.

$$
\frac{d \log _{10}\left\langle\Delta r^{2}(\tau)\right\rangle}{d \log _{10} \tau}=\frac{R_{m}^{2}}{\left\langle\Delta r^{2}(\tau)\right\rangle}\left[C \delta\left(\frac{\tau}{\tau_{m}}\right)^{\delta}+C^{\prime}\left(\frac{\tau}{\tau_{m}}\right)\right]
$$

In Eq. (4) we defined this quantity to be equal to the stretching index $\nu$ at $\tau=\tau_{m}$. Using this gives the second relationship: $\nu=C \delta+C^{\prime}$. Finally, from Eq. (4) it is also clear that $\left\langle\Delta r^{2}\left(\tau_{m}\right)\right\rangle \propto \tau^{\nu}$ at $\tau=\tau_{m}$. Thus, $\nu=\delta$.

Use of these relationships gives $C^{\prime}=\nu(1-C)$ and $C^{\prime \prime}=(1-C)(1-\nu)$, with which Eq. (10) can be expressed as follows:

$$
\begin{aligned}
\left\langle\Delta r^{2}(\tau \geqslant\right. & \left.\left.\tau_{m}\right)\right\rangle=\left[C\left(\frac{\tau}{\tau_{m}}\right)^{\nu}+\nu(1-C)\left(\frac{\tau}{\tau_{m}}\right)\right. \\
& +(1-\nu)(1-C)] R_{m}^{2} .
\end{aligned}
$$

This can now be fitted to the MSDs using the known values of $\nu, \tau_{m}$, and $R_{m}$, with $C$ as the only free parameter. Typical least-squares fits are included in Fig. 1 and the values of $C$ are shown in Fig. 8. A number of consistency checks are now performed:

(i) The algebraic form of the VAF with the following expression for the scaling time:

$$
\tau_{f}=\left[\frac{\tau_{m}^{\nu}}{C \nu(1-\nu) R_{m}^{2}}\right]^{1 /(\nu-2)}
$$

is obtained by equating the second derivative of Eq. (12) with Eq. (8). Figures 6 and 7 show the values for $2-\nu$ and $\tau_{f}$, respectively. These confirm that $\mu(=2-\nu)$ varies from $\mu \approx 3 / 2$ at $\varphi=\varphi_{f}$, to $\mu \approx 2$, at $\varphi=\varphi_{g}$, and that $\log _{10}\left(\tau_{f}\right)=-1.14 \pm 0.07$, compared with $\log _{10}\left(\tau_{f}\right)=-1.2 \pm 0.3$ obtained above by directly fitting power laws to the VAF, shows no systematic variation with $\varphi$.

(ii) At long times the term linear in $\tau$ in Eq. (12), i.e., the term corresponding to diffusion, dominates for delay times $\tau>\tau_{x}$, where

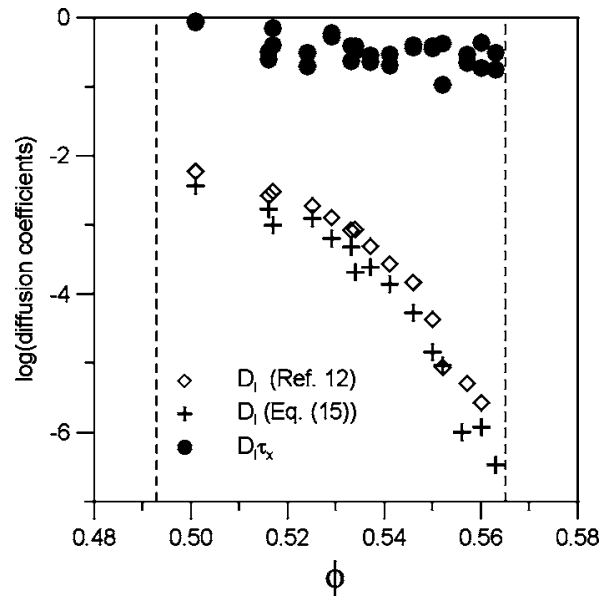

FIG. 9. Long-time self-diffusion coefficients from previous measurements compared with those calculated from Eq. (15).

$$
\tau_{x}=\left(\frac{C}{\nu(1-C)}\right)^{1 /(1-\nu)} \tau_{m}
$$

This is the delay time where the self-ISF, given by Eq. (2), crosses over from a stretched exponential to an exponential function of $\tau$. For $\varphi=0.533$, for example, where we find $C$ $=0.8$ and $\nu=0.33, \tau_{x} \approx 42 \tau_{m}$. Increasing the measurement time sufficiently so that the term nonlinear in $\tau$ reduces to a few percent of the total, results in the onset of crystallization. Thus the diffusive limit, which must be attained in the limit $\tau \rightarrow \infty$ for a system in thermodynamic equilibrium, is not attainable in the metastable suspension because it can only equilibrate by crystallizing.

Having said this does not prevent us from taking the limit, $\tau \rightarrow \infty$, in the right-hand side of Eq. (12). Given that $\nu<1$, one arrives at the following expression for the long-time selfdiffusion coefficient:

$$
D_{l}=\frac{\nu(1-C) R_{m}^{2}}{\tau_{m}}
$$

In Fig. 9 the values of $D_{l}$ obtained by this procedure are compared with those obtained directly from the long-time exponential decay of the self-ISF $[12,17]$. The differences, bordering experimental uncertainty, are small but consistent. However, in view of the difficulty, mentioned above, in actually reaching the long-time diffusive limit in practice, especially at the elevated volume fractions considered here, we regard the values of $D_{l}$ obtained by means of Eq. (15) as the better estimates. The product, $D_{l} \tau_{x}$, is also shown in Fig. 9. Given that $D_{l}$ and $\tau_{x}$ both characterize the long-time exponential decay of the self-ISF it comes as no surprise that $D_{l} \tau_{x}$ shows comparatively little systematic variation with $\varphi$.

One also sees from Fig. 7 that $\tau_{x}$ is quantitatively consistent with the ratio $\tau_{m} / \tau_{c}$. So there appears to be a symmetry about the point $\left(R_{m}^{2}, \tau_{m}\right)$ : the time required for memory of the collective processes, exposed at $\tau_{m}$, to be lost corresponds to the number of diffusive encounters involved in these processes. This loss of memory leads to Markovian exploration of configuration space, characterized by the coefficient $D_{l}$. However, this form of exploration results from the central 
limit theorem whose efficacy, in turn, derives from the fact that the linear dimension of the scattering volume is much larger than the extent of spatial correlation in the (amorphous) suspension. (Of course the ensemble average over phases, $\mathbf{q} \cdot \Delta \mathbf{r}$, and the spatial limit, $q \rightarrow 0$, are implicit.) So, in the context of the present analysis at least, the existence of $D_{l}$ for the metastable suspension does not imply that, on increasing $\tau$, the displacements of an arbitrarily chosen particle become Markovian. The fact that the long-time diffusive limit has been obtained from Eq. (12), while the first term is retained, indicates that the structures responsible for the stretching of the MSD, exposed at $\tau_{m}$, remain intact during the course of the experiment, i.e., for as long as metastability or quasistationarity of the suspension prevails.

Since an expression for the MSD cannot be obtained analytically where the VAF obeys a stretched exponential, consistency checks analogous to the above cannot be applied to the thermodynamically stable suspension. However, in this case, $D_{l}$ is found to be proportional to $R_{m}^{2} / \tau_{m}$ [20]. So, for the metastable suspension, the long-time diffusion coefficient is weighted by an extra factor, $\nu(1-C)$, that decreases with $\varphi$, a factor which, as we have just seen, characterizes structures that do not dissipate on time coarse graining. Reliance on, and ensuing interpretation of, the index $\nu$ is only possible so long as the time scales of thermal kinetics and density fluctuations are well separated, i.e., so long as stretching of the MSD is not compounded by the scalar, or Gaussian, thermal kinetics.

\section{B. Dynamical heterogeneity}

The Lorentz gas [14] is perhaps the best known model whose asymptotic dynamics is characterized by a negative algebraic decay of the VAF, $\propto-\tau^{-(1+d / 2)}$, where $d$ is the dimension of the system. In this model the positions of the targets are fixed and consequently momentum is not conserved. Of course one may argue that the suspension is not even remotely similar to a Lorentz gas, particularly one having dimension less than three. However, there is a statistical equivalence of this model and that aspect of the nonMarkovian particle excursions manifested by the negative algebraic VAF. Translating this into dynamical analogy suggests that packing constraints in the metastable suspension are such that some particles do not respond to the thermally activated momentum currents in the suspending liquid, or they are to some extent at least impaired from doing so. Of course the momentum current cannot be read directly from these DLS data. But one can ask, nonetheless, whether or not the observed particle displacement statistics are compatible with the known manifestations of momentum conservation.

The reasoning used in Ref. [13] to arrive at the conclusion that momentum conservation is violated in the metastable suspension runs as follows: Let the function $-f(\tau)$ express a particular (non-Markovian) contribution to the slow decay of the VAF in the experimental time window. Then, as $\tau \rightarrow 0$, the corresponding particle motions can couple to diffusing shear modes in the suspending liquid, and satisfy the condition $Z(0)>0$, only if $f(\tau)$ is a weaker function of $\tau$ than $\tau^{-3 / 2}$. Of course, this (positive) power law will be recognized as the classical hydrodynamic "tail" that manifests the presence of, or response to, diffusing transverse momentum currents [22]. Accordingly, the displacements observed for the thermodynamically stable suspension, expressed by the stretched exponential decay in Eq. (7), are consistent with diffusing shear modes. Indeed, with the reasoning adopted here one arrives at the predicted value for the amplitude of the classical hydrodynamic "tail" [13]. However, that part of the VAF given by the power law, Eq. (8), with the index, $\mu>3 / 2$, is not compatible with normal viscous flow. From this we infer that, in the metastable suspension, the movements of some particles are so constrained that their ability to respond to diffusing shear modes in the suspending liquid is impaired. And, in analogy with the Lorentz gas, the presence of these trapped particles effects a negative feedback on the direction of motion of the mobile particles. This feedback is independent of the degree of damping of the particles' motion. We propose, therefore, that the consequential enhancement of the persistence of the VAF results in the crossover, seen in Fig. 5(c), from a stretched exponential to an algebraic function of the delay time.

Although, as we have seen, the long-time diffusion coefficient exists, the structural constraint on diffusing shear modes means that the viscosity, in the limit of zero shear rate, does not. The implied departure from the reciprocity of the diffusion coefficient and the Newtonian viscosity, expressed by the Stokes-Einstein equation, has been established in other work [23] by analyses of experimental dynamic viscosities and diffusivities of metastable hard-sphere suspensions.

The picture in which some particles are trapped in clusters with the remaining more mobile particles necessarily confined to the interstitial channels is familiar to us from numerous microscopic studies [8-11]. To be confident that these clusters are not crystal nuclei, we appeal to kinetic studies of similar systems. First, between $\varphi_{f}$ and $\varphi_{g}$ the amplitude of the VAF power law varies by only a factor of 10 . Kinetic experiments, on the other hand, show that nucleation rate densities vary by three to four orders of magnitude over the same range $[24,25]$. If crystallites were the cause of the velocity reversals observed here, one might expect the variation in the amplitude of the power law to be comparable with the many orders of magnitude found for the variation of the nucleation rate density.

Second, we note that the negative power law sets in at, or very near to, $\varphi_{f}=0.494$. By contrast, kinetic studies of the growth of crystals show that for volume fractions just above freezing, $0.494<\varphi<0.51$, no homogeneous crystallization is evident even on time scales of $10^{5} \mathrm{~s}$, two orders of magnitude longer than the measurements made here [24,25]. The fact that the algebraic decay of the VAF is already clearly evident at $\varphi=0.5$, where homogeneous crystallization is never observed, conclusively demonstrates that the presence of crystallites cannot explain these results.

Another clue about this heterogeneous dynamics and, in particular, the qualitative distinction between the thermodynamically stable and metastable suspensions, derives from the stretching index $\nu$. From Fig. 2 one notes that $\nu=1 / 2$ at $\varphi_{f}$. So that aspect of the particle movements exposed at $\tau_{m}$ is that for which the MSD grows in proportion to $\tau^{1 / 2}$ or, 
equivalently, the VAF in proportion to $-\tau^{-3 / 2}$. Another model for which the VAF decays in just this manner comprises (diffusing) particles subject to the single file condition (SFC) $[15,26]$. The asymptotic result for this case is

$$
Z_{\mathrm{SF}}(\tau)=-\frac{S}{2} \sqrt{\frac{D}{\pi}} \tau^{-3 / 2}
$$

Here $D$ is the diffusion coefficient and $S$ is the average gap between the particles in the file. Equating Eq. (16) and Eq. (8), with $\mu=3 / 2$, gives

$$
\tau_{f}=\left(\frac{S^{2} D}{4 \pi}\right)^{1 / 3} .
$$

A consistency check is readily performed. Substituting into the right-hand side of Eq. (17) $S=R_{c}\left(\varphi_{f}\right)=0.09$ [from Eq. (6)] and $D=D_{s}\left(\varphi_{f}\right)=0.17$ [from the data] one obtains $\tau_{f}$ $=0.05$ (or $\log _{10} \tau_{f}=-1.3$ ). This value for the scaling time is consistent with that obtained from the power-law analysis of the VAF in Secs. III and IV A. Thus, the non-Markovian movement exposed at the freezing volume fraction can be described entirely by the asymptotic displacement statistics of a percolating file of particles. Particles thus constrained are, in view of the discussion above, at the threshold of their ability to couple to diffusing shear modes.

Values of $1, \frac{1}{2}$, and 0 for the index $\nu$, respectively, indicate diffusing particles, particles subject to the SFC, and particles that are trapped. So, in view of Fig. 2 the point, $\varphi_{f}$, of the first order transition acts as a dynamical pivot where there is an exchange of diffusing and trapped particles. For $0 \leqslant \varphi$ $\leqslant \varphi_{f}$ where, according to Fig. $2,1 \geqslant \nu \geqslant 1 / 2$, the particles' movements can be described by a nonlinear combination of diffusing particles and particles subject to the SFC. Again, it must be stressed that this is what is exposed at $\tau_{m}$. For much shorter delay times, say $\tau \approx \tau_{c}$, excluded volume effects are not exposed and all particles appear to diffuse. The coupling of particles subject to the SFC and diffusing particles means that for delay times much longer than $\tau_{m}$, where all memory of excluded volume effects has been lost, all particles are also seen to diffuse.

In the metastable suspension, $\phi_{f} \leqslant \varphi \leqslant \varphi_{g}$, where $1 / 2$ $\geqslant \nu \geqslant 0$, the dynamics can be described by an interdependent combination of particles subject to the SFC and particles that are localized-respectively, contributing fractions $2 \nu$ and 1 $-2 \nu$ to the MSD. For delay times $\tau \geqslant \tau_{m}$, the effects of thermal forces have been coarse grained to the noise floor. So there no effective randomizing process to dislodge the trapped particles. At $\varphi_{g}$ the system no longer supports a percolating file and all particles are localized. While all the particles are trapped at $\varphi_{g}$, the root-mean-squared displacement, $R_{m}$, at $\tau_{m}$ is approximately 10 times the average gap, $R_{c}$, between the particle surfaces. So the dynamical heterogeneity is frozen in. This contrasts the situation in the crystalline phase where all particles are confined to identical WignerSeitz cells and $R_{m} \approx R_{c}$ [27].

\section{CONCLUSION}

We propose that the dynamical heterogeneity identified in microscopic studies is simply a manifestation of the fact that, when viewed over a given delay time, the movements of some of the particles in a suspension are blocked by other particles in their paths. Blockages so experienced are no different from those experienced by particles subject to the single file condition. This aspect of the collective dynamics is exposed in the MSD of a colloidal suspension because the separation of time scales is such that the scalar thermal kinetics do not compound stretching of the MSD. For suspensions in thermodynamic equilibrium such blockages are dissipated by Brownian motion. In the metastable suspension the packing constraints are so severe that Brownian forces are no longer able to dissipate all of the blockages-some particles remain trapped in clusters. Then for $\tau \gg \tau_{c}$, i.e., for delay times far greater than those which characterize the average interval between diffusive encounters, when the local motions of the trapped particles contribute no further to the MSD, the clusters appear rigid, not unlike the grains in a granular fluid. And in their inability to respond to the thermal agitation, the grains act as momentum sinks.

If the grains, or more specifically the particles within them, cannot dissipate the thermal stresses applied by the suspending liquid then, under such action the grains may pile up and percolate the system. Here, as in Ref. [11], one arrives at the structures that may support low frequency elastic modes. So, the presence of trapped particles allows diffusing shear modes to be exchanged for (low frequency) propagating shear modes. In addition, the long-time diffusion coefficient is reduced, relative to that of the thermodynamically stable suspension, by a factor that characterizes the trapped particles.

\section{ACKNOWLEDGMENT}

We are grateful to Salvy Russo for valuable comments on the manuscript.
[1] P. N. Pusey and W. van Megen, Nature (London) 320, 340 (1986).

[2] P. N. Pusey and W. van Megen, Phys. Rev. Lett. 59, 2083 (1987)

[3] S. I. Henderson, T. C. Mortensen, S. M. Underwood, and W. van Megen, Physica A 233, 102 (1996); S. I. Henderson and W. van Megen, Phys. Rev. Lett. 80, 877 (1998).

[4] H. J. Schöpe, G. Bryant, and W. van Megen, Phys. Rev. E 74,
060401(R) (2006).

[5] S. R. Williams, I. K. Snook, and W. van Megen, Phys. Rev. E 64, 021506 (2001).

[6] S. R. Williams, G. Bryant, I. K. Snook, and W. van Megen, Phys. Rev. Lett. 96, 087801 (2006).

[7] F. Sciortino and P. Tartaglia, Adv. Phys. 54, 471 (2005), and references therein.

[8] M. M. Hurley and P. Harrowell, Phys. Rev. E 52, 1694 (1995); 
W. Kob, J. Phys.: Condens. Matter, 11, R85 (2000), and references therein.

[9] S. C. Glotzer, J. Non-Cryst. Solids 274, 342 (2000), and references therein.

[10] W. K. Kegel and A. van Blaaderen, Science 287, 290 (2000); E. R. Weeks et al., ibid. 287, 627 (2000).

[11] J. C. Conrad, P. P. Dhillon, E. R. Weeks, D. R. Reichman, and D. A. Weitz, Phys. Rev. Lett. 97, 265701 (2006).

[12] W. van Megen, T. C. Mortensen, and G. Bryant, Phys. Rev. E 72, 031402 (2005).

[13] W. van Megen, Phys. Rev. E 73, 020503(R) (2006).

[14] M. H. Ernst and A. Weyland, Phys. Lett. A 34, 39 (1971).

[15] T. E. Harris, J. Appl. Probab. 2, 323 (1965); H. van Beijeren, K. W. Kehr, and R. Kutner, Phys. Rev. B 28, 5711 (1983).

[16] W. van Megen and S. M. Underwood, J. Chem. Phys. 91, 552 (1989).

[17] W. van Megen, T. C. Mortensen, S. R. Williams, and J. Müller, Phys. Rev. E 58, 6073 (1998).

[18] G. Bryant, S. R. Williams, Q. Lin Mac, I. K. Snook, E. Perez, and F. Pincet Phys. Rev. E 66, 060501(R) (2002).
[19] J. P. Hansen and I. R. McDonald, Theory of Simple Liquids (Academic, London, 1986).

[20] W. van Megen, Phys. Rev. E 73, 011401 (2006).

[21] This caveat, $\mu=2$, is not necessarily excluded by the data at $\varphi \approx \varphi_{\mathrm{g}}$ where $\mu \approx 2$. Its implication, a contribution to the MSD proportional to $\log _{10}(\tau)$, was recently identified in an analysis of these data by mode coupling theory [M. Sperl, Phys. Rev. E 71, 060401(R) (2005)]. This issue and a more detailed comparison of these data with the mode coupling theory are the subjects of a future publication.

[22] B. J. Alder and T. E. Wainwright, Phys. Rev. A 1, 18 (1970).

[23] D. Bonn and W. K. Kegel, J. Chem. Phys. 118, 2005 (2003).

[24] J. L. Harland, S. I. Henderson, S. M. Underwood, and W. van Megen, Phys. Rev. Lett. 75, 3572 (1995).

[25] H. J. Schöpe, G. Bryant, and W. van Megen, Phys. Rev. Lett. 96, 175701 (2006).

[26] Q.-H. Wei, C. Bechinger, and P. Leiderer, Science 287, 625 (2000).

[27] Z. Cheng, J. Zhu, W. B. Russel, and P. M. Chaikin, Phys. Rev. Lett. 85, 1460 (2000). 